\title{
Professor Wang Xisheng's Thoughts on Tumors and "Fu Yuan Fu Heng Method" for the Treatment of Malignant Tumors
}

\author{
Zhengyang Chen ${ }^{1}$, Xisheng Wang ${ }^{2 *}$ \\ ${ }^{1}$ Graduate School of Shaanxi University of Traditional Chinese Medicine, Xianyang 712000, China \\ ${ }^{2}$ Affiliated Hospital of Shaanxi University of Traditional Chinese Medicine, Xianyang 712000, China
}

\begin{abstract}
According to the report of the National Cancer Center, there are about 4.5 million new cancer patients and 3 million deaths in China every year. How to effectively implement a comprehensive system of prevention and control measures and reduce the incidence rate of malignant tumors in the country, so that the mortality rate of cancer is effectively controlled is a problem that needs to be solved urgently. In particular, it is worth mentioning that in China, the addition of traditional Chinese medicine in the treatment of malignant tumor plays an important role in improving the therapeutic effect, reducing the toxic and side effects of comprehensive treatment, improving the quality of life, and "survival with tumor" of end-stage cancer patients. Professor Wang Xisheng has been engaged in the field of traditional Chinese medicine in the treatment of cancer for more than 40 years. In his long-term clinical practice, he has accumulated rich experience in the prevention and treatment of malignant tumors. This article introduces Professor Wang's thinking on cancer in clinical practice and the "Fu Yuan Fu Heng method" in the treatment of malignant tumors for reference.
\end{abstract}

Key words: Thinking about tumor; Fuyuan Fuheng Decoction; Cancer treatment

Publication date: May, 2021; Publication online: 31 May, 2021

*Corresponding author: Xisheng Wang, 1028945212@qq.com

\section{Some thoughts on tumor}

\subsection{What is a tumor?}

Modern medicine believes that tumor is a new organism formed by abnormal gene expression and abnormal cell proliferation caused by a variety of tumorigenic factors ${ }^{[1]}$. After malignant tumor cells form, they are no longer regulated by the normal regulatory mechanism of the human body, cause damage to normal tissues and organs, and seriously endanger human health. The earliest description of "tumor" in traditional Chinese medicine can be traced back to Oracle Bone Inscriptions in the Yin and Shang Dynasties. Due to the deficiency of Qi, blood stasis, and accumulation of symptoms, the function of viscera is out of balance; Or by the body movement of dereliction of duty, phlegm condensation, gas and phlegm accumulation does not disperse; The interaction of blood stasis, phlegm turbidity and pathogenic toxin will lead to cancer. Professor Wang thought: Tumor is a natural thing, and its survival depends on the human body. Since tumor is a kind of existence, philosophically speaking, "existence is reasonable", it seems that there should be a reason for its existence, but human beings have not found it, let alone observed and studied it. What exactly is a tumor for? Why does it exist? Is cancer always harmful to human body? Why do we have the idea of "survival with tumor"?

\subsection{Why do you get tumors?}

Why do people get tumors? Is it related to population, age, gender, status, poverty and wealth? Based on current population with cancer, it is shown that etiology of cancer is often unknown, and the incidence is random. The method theory of traditional Chinese medicine is rooted in Chinese 
culture $^{[3]}$. Inspired by traditional cultural ethics, Professor Wang tried to explain why people get tumors by using the nature, instinct, disposition and attributes of all natural things. Professor Wang often gives examples. This is the nature of human beings. Fish are not easily affected by dampness. It seems that cancer patients themselves have the possibility of suffering from cancer. It can also be said that this kind of people suffering from cancer is inevitable, which is determined by nature, nature, disposition and destiny. Modern genetic medicine and preventive medicine involved in genetic engineering, gene testing confirmed that genetic mutation is directly related to the occurrence of some diseases, also confirmed the nature of suffering from a certain disease $\mathrm{e}^{[4]}$.

\subsection{How to face cancer?}

Professor Wang thinks that since cancer is an objective existence, we must face it calmly when facing diseases like cancer. It is inevitable for people to get sick. Some people are born disabled, which does not affect their normal life. Correct understanding of the disease, reducing the ideological burden, strengthening nutritional care, maintaining a good attitude, increasing rest time, maintaining moderate exercise, timely symptomatic treatment after symptoms are helpful for the treatment and recovery of tumor.

\subsection{How to treat tumor?}

Benefiting from the continuous development of basic disciplines and biological science and medical technology, significant progress has been made in the research of tumor etiology, pathogenesis, treatment and prevention, and new drugs, new technologies and new therapies have come out one after another ${ }^{[5-6]}$. Professor Wang believes that although there are many treatments, the mortality rate is still high. The difficulty of tumor treatment lies in that each treatment has its limitations. It is impossible to cure the tumor by a single method. Although the treatment has benefits, it should be stopped when it does too much harm to the body. For cancer treatment is not to eliminate tumor cells as the primary goal, but to promote appropriate adjustment to mobilize the body's ability of self-recovery, self-resistance and self-regulation.

\section{Fuyuan Fuheng Decoction in the treatment of}

\section{malignant tumor}

Human is a product of nature. As a part of nature, Professor Wang thinks that human body has wonderful structure and perfect function ${ }^{[7]}$. Corresponding to the external heaven, earth and human beings, they have the same Qi, isomorphism and law with nature. They conform to nature, adapt to nature, make use of nature, integrate the internal form and spirit, self-adjust, self-renew and self-repair, conform to the time, and live to the age of heaven ${ }^{[8]}$. According to Tao Te Ching, the best state of human existence is harmony. The balance of Qi and blood, yin and Yang, viscera and body fluid is particularly important to maintain health. Professor Wang believes that Yuanqi can adjust the balance of viscera, Qi and blood, body fluid, meridians and Qi mechanism, maintain the balance and coordination of viscera and meridians function, harmonize qi and blood, enrich body fluid, and keep Qi mechanism up and down. Traditional Chinese medicine can help to alleviate the pain of patients, restore the regulatory role of vitality, regulate and conform to the homeostasis of human body, promote the ability of self-healing and self-defense of human body, and make tumor and human coexist harmoniously. Thus, the idea of "Fu Yuan Fu Heng" runs through the whole process of tumor treatment and rehabilitation. "Fu Yuan" is to improve the "deficiency of vital energy" of cancer patients, and "Fu Heng" is to restore the balance of patients by supporting vital energy.

\section{References}

[1] Gong ZF. Mechanism and prevention of malignant tumor formation[J]. Journal of Shandong Medical University: Social Science Edition, 1995 (2): 10-12.

[2] Chen BX. Prevention and treatment of tumor with traditional Chinese medicine [J]. Journal of Tongji University: Medical Edition, 2002, 23 (2): 104-105.

[3] Jiang LS. Traditional Chinese medicine is rooted in Chinese traditional culture $[\mathrm{J}]$. Jiangxi traditional Chinese medicine, 2007, 38(9): 1.

[4] Fan LH, Liu ML, Liu TF. Research and application of genetic engineering animal model of human disease [J]. Review of medicine, 2009, 15(7): 1009-1012.

[5] Xing XY, Wang XC, He W. Progress of tumor immunotherapy and drug research and development $[\mathrm{J}]$. Journal of China Pharmaceutical University, 2021, 52 (1): $10-19$

[6] Wang XM, Gu XS. Multidisciplinary treatment of malignant tumor [J]. Anhui medicine, 1990 (4): 3-4.

[7] Tian SD, Chen XY. Characteristics and advantages of traditional Chinese medicine in the treatment of 
malignant tumors $[\mathrm{J}]$. Journal of Beijing University of traditional Chinese medicine (Clinical edition of traditional Chinese Medicine), 2019, 26(2): 8-17.

[8] Zheng P. Conform to the law of natural change, reflect the characteristics of traditional Chinese medicine nursing $[\mathrm{J}]$. Contemporary medicine, 2009, 15 (3):
89-90.

[9] Shi S, Ji XY, Hu YH, et al. The influence of Tao De Ching on the formation of TCM diagnosis and treatment thinking $[\mathrm{J}]$. Tianjin traditional Chinese medicine, 2020, 37(10): 1147-1149. 